\title{
Expression of Hepatocyte Transporters and Nuclear Receptors in Children With Early and Late-Stage Biliary Atresia
}

\author{
HUEY-LING CHEN, YU-JUNG LIU, HUI-LING CHEN, SHANG-HSIN WU, YEN-HSUAN NI, MING-CHIH HO, HONG-SHIEE LAI,
} WEN-MING HSU, HONG-YUAN HSU, HUI-CHIH TSENG, YUNG-MING JENG, AND MEI-HWEI CHANG

\author{
Departments of Pediatrics [H.C., Y.L., S.W., Y.N., H.H., H.T., M.C.], Primary Care Medicine [H.C., H.H.], Surgery [M.H., H.L., W.H.], \\ and Pathology [Y.J], National Taiwan University College of Medicine and Hospital, Taipei, 10002 Taiwan; Hepatitis Research Center
} [H.C.], National Taiwan University Hospital, Taipei, 10048 Taiwan

\begin{abstract}
To investigate how the liver adapts to chronic obstructive cholestasis, liver samples from infants with early- and late-stage cholestasis were analyzed for changes in the levels of hepatocyte transporters and nuclear receptors. At early-stage cholestasis, most canalicular transporters and sinusoidal uptake transporters were downregulated, including bile salt export pump (BSEP, ABCB11), multidrug resistant protein 3 (MDR3, ABCB4), multidrug-resistant associated protein 2 (MRP2, ABCC2), sodium-dependent taurocholate cotransporting polypeptide (NTCP, SLC10A1), organic anion transporter (OATP, SLCO1A2), and nuclear receptor farnesoid X receptor (FXR, NR1H4). At late-stage cholestasis, FXR-BSEP levels returned to normal, MDR3 and MDR1 (ABCB1) were upregulated, and MRP-2 was downregulated. In addition, alternative sinusoidal efflux transporters, organic solute transporter alpha/beta $(\mathrm{OST} \alpha / \beta)$ and MRP4 were upregulated, and pregnane X receptor (PXR, NR1I2) levels decreased. Cytochrome enzyme P450 7A1 was markedly downregulated at both early and late-stage cholestasis. An analysis of the long-term prognosis of 18 patients revealed lower PXR and constitutive androstane receptor (CAR, NR1I3) levels in the poor prognosis group. In conclusion, at long-term cholestasis, hepatocyte bile efflux was through sinusoidal and canalicular transporters, with FXR-BSEP levels maintained and PXR downregulated. Low PXR and CAR levels were associated with poor prognosis. (Pediatr Res 63: 667-673, 2008)
\end{abstract}

$\mathrm{O}$ bstructive cholestasis, caused by biliary tract stones, external tumor compression, or congenital biliary anomalies, is a common devastating liver dysfunction, resulting in liver toxicity, cirrhosis, and eventually hepatic failure. Therapy for obstructive cholestasis is mainly surgical (1). However, those who cannot undergo corrective surgery, receive medical treatments, which have limited efficacy and may be detrimental to overall health (2).

Clinical response to acute and chronic obstructive cholestasis differs. Acute obstructive cholestasis results in rapidly increasing bilirubin levels and deterioration of liver function. Animals with common bile duct ligation rapidly progressed to liver cirrhosis and died in a few weeks (2). In chronic cholestasis such as biliary atresia (BA), the bilirubin levels and

Received November 5, 2007; accepted January 30, 2008.

Correspondence: Mei-Hwei Chang, M.D., Department of Pediatrics, National Taiwan University Hospital, 7F, No. 7, Chung-Shan South Road, Taipei 100, Taiwan, China; e-mail: changmh@ntu.edu.tw

This study was supported by grant from National Science Council, Taiwan (NSC-942314-B-002-247 and NSC-95-2314-B-002-056), and partly by Liver Disease Prevention \& Treatment Research Foundation, Taiwan. transaminase levels remained at a stable level until very late stage. BA, as the leading cause for pediatric liver transplantation, represents a unique human model of chronic cholestatic liver diseases and the best paradigm for studying acute and chronic cholestatic responses. Kasai portoenterostomy performed before 2 mo of age provides curative or palliative biliary drainage and prolongs patient survival. However, some patients continue to have progressive obstructive cholestasis, with survival up to 0.6 to 3 y with little bile drainage $(3,4)$. Under such circumstances, it is highly possible that the liver adapts to protect hepatocytes from the toxic effect of rapidly accumulating bile acids and other metabolites.

Recent studies of the molecular mechanisms of bile physiology have provided better understanding of the pathophysiology for various cholestatic liver diseases. The sinusoidal transporters, sodium-taurocholate cotransporting polypeptide (NTCP, SLC10A1) and organic anion transporter (OATP, SLCO1A2) mediate uptake of bile acids and organic solutes; cytochrome P450 (CYP) enzymes hydroxylize and metabolize bile acids; and the canalicular export pumps, bile salt export pump (BSEP, ABCB11), multidrug-resistant gene 3 (MDR3, ABCB4), multidrug-resistant associated-protein 2 (MRP2, ABCC2) mediate bile acids, phospholipids, and bilirubin export, respectively $(5,6)$. Some of the genetic defects result in progressive familial intrahepatic cholestasis. Several nuclear receptors have been found to be important in regulating hepatocellular transport and metabolic functions. The key nuclear receptors mediating bile acid homeostatsis include farnesoid X receptor (FXR, NR1H4), short heterodimeric partner (SHP, NR0B2), pregnane $X$ receptor (PXR, NR1I2), and constitutive androstane receptor (CAR, NR1I3) (7-13). New therapeutic agents for cholestatic liver disease aiming at modulating the nuclear receptors is actively being investigated (14).

It is important to understand the changes and alternative pathways in the human liver in response to cholestasis before the proposed treatment could be applied in clinical settings.

\footnotetext{
Abbreviations: BA, biliary atresia; BSEP, bile salt export pump; CAR, constitutive androstane receptor; CYP, cytochrome enzyme P450; FXR, farnesoid X receptor; MDR, multidrug resistant protein; MRP, multidrug resistant associated-protein; NTCP, sodium-dependent taurocholate cotransporting polypeptide; OATP, organic anion transporter; OLT, orthotopic liver transplantation; OST $\boldsymbol{\alpha} / \boldsymbol{\beta}$, organic solute transporter alpha/beta; PXR, pregnane $\mathrm{X}$ receptor; SHP, short heterodimeric partner
} 
Previous studies have investigated the changes of a few key canalicular transporters in adult patients (15-18). However, different forms of cholestasis may exhibit distinct transporter regulation. Data regarding pediatric liver diseases is lacking in this aspect. We investigated the expressions of hepatocyte transporters and nuclear receptors comprehensively in patients with early-stage and late-stage BA. These data provide evidence of how human liver responds to biliary obstruction to ameliorate accumulating bile acid and metabolites injury at different stages of the disease.

\section{METHODS}

Patient samples. BA was diagnosed by clinical symptoms, liver biopsy, and confirmed by intraoperative cholangiography. Liver samples from eight patients with BA at the age of 2 mo $(64 \pm 17$ d) during Kasai operation represented early-stage obstructive cholestasis. Liver samples from eight BA patients aged 8 to $15 \mathrm{mo}(10.50 \pm 3.12 \mathrm{mo})$ receiving orthotopic liver transplantation (OLT) represented late-stage cholestasis. The patients in late-stage group had all received Kasai operation at 2 mo of age but did not obtain patent bile flow after the surgery. They developed progressive liver cirrhosis and liver failure that required subsequent OLT at young age.

Pediatric control samples were collected from eight patients with noncholestatic liver diseases, including patients with hepatoblastoma (nontumor part), hepatitis, and inherited metabolic diseases. Their ages were 6 to $17 \mathrm{mo}$ (12.01 $\pm 3.86 \mathrm{mo}$ ). Four adult samples (aged 20-40 y) from living-donor liver were used for adult control in the immunofluoresecent study for localization of canalicular transporters.

To further investigate the expression of canalicular transporters at earlystage of disease, and to correlate with long-term prognosis in patients with BA, a total of 18 patients with BA with liver samples obtained at Kasai operation were analyzed. Nine patients designated as good prognosis had good bile flow after Kasai operation. The stool color turned from pale to yellowish after the operation. The serum bilirubin level returned to normal, with total bilirubin level maintained below $2 \mathrm{mg} / \mathrm{dL}$ for at least $2 \mathrm{y}$. They were followed up for 23 to $118 \mathrm{mo}$. Another nine patients were designated as poor prognosis. They had progressive cholestasis after the Kasai operation, with persistently elevated serum bilirubin levels. Within 2 y of follow-up, they were either expired, received OLT, or had advanced cholestasis/cirrhosis awaiting OLT.

Liver specimens from needle biopsy or surgical biopsy were snap-frozen, and stored in liquid nitrogen for RNA and bile acid analysis, or embedded in O.C.T. (Tissue-Tek, Sakura) and stored in a $-80^{\circ} \mathrm{C}$ freezer for tissue sections and staining. Informed consents were obtained from the parents. This study has been approved by the Institutional Review Board of National Taiwan University.

Quantitative reverse transcription polymerase chain reaction. Total RNAs were extracted from frozen liver by RNAeasy kit (Qiagen $\mathrm{GmbH}$, Hilden, Germany), and was reverse-transcripted with 200 pmol of random hexamer (Invitrogen Life Technologies, Breda, Netherlands) and reverse transcriptase (SuperScript III, Invitrogen Life Technologies). With the cDNA (cDNA) obtained, PCR reaction was performed using the Taqman system in a PRISM 7900 HT Sequence Detection System (Applied Biosystems, Foster City, CA). Aliquots (5 ng) of total RNA were used for each reaction. The primers and probes used were obtained from TaqMan ${ }^{\circledR}$ Gene Expression Assays (Applied Biosystems), including TATA-box biding protein, MDR1,
MDR3, MRP2, MRP3, MRP4, NTCP, OATP1B1, OATB1B3, organic solute transporter alpha (OST $\alpha)$, organic solute transporter beta (OST $\beta)$, CYP7A1, CYP3A4, SHP, FXR, PXR, and CAR. BSEP primers and probes were designed using published human cDNA sequences in Genbank database with Primer Express software v1.5 (Applied Biosystems). All reactions were run in duplicate. Data were analyzed with Sequence Detector v2.2.2 software (Applied Biosystems).

The expression levels of transporter genes relative to the housekeeping gene, TATA-box biding protein were calculated using the Standard Curve Methods (ABI PRISM User Bulletin 2). The mean levels of gene expression in early- and late-stage BA liver were compared with the levels in pediatric control liver, respectively.

Immunofluorescent staining. Frozen tissue sections $(5-7 \mu \mathrm{m})$ were cut in cryostat and placed on silanized slides (DAKO), then fixed in cooled acetone. The antibodies used included a polyclonal antibody against human BSEP (1:500) (19), MAb for MDR1 (ABCB1, 1:500, Sigma Chemical Co., Saint Louis, Missouri), MRP2 $\left(\mathrm{M}_{2} \mathrm{III}-6 ; 1: 100\right)$, and MDR3 ( $\left.\mathrm{P}_{3} \mathrm{II}-26 ; 1: 100\right)$ from Alexis Biochemicals (San Diego, CA). A polyclonal human OST $\beta$ antibody (hOSTB1; $1: 100$ ) was kindly provided by Dr. Nazzareno Ballatori. Fluorescent conjugated secondary antibody (Alexa Fluor 594 and Alexa Fluor 488, Molecular Probes) was used. The images were obtained using image capture system (Leica DMRD, CoolSNAP) and Nikon C1 confocal microscope and processed using Adobe Photoshop CS2 (Adobe).

Bile acid analysis. Serum total bile acids were measured using the bile acids assay kit (DIAZYME Laboratories, Dan Diego, CA). For liver bile acid measurement, total bile acid was extracted by $75 \%$ ethanol in $50^{\circ} \mathrm{C}$ for $2 \mathrm{~h}$. After centrifugation, the supernatant was measured according to manufacturer's instructions.

Statistics. The differences of mean expression levels between groups were compared with $t$ test. A $p$ value $<0.05$ was considered to be significant. Kaplan Maier survival analysis was used to compare the patient survival in different groups. Statistics were performed using SPSS 12.0 (SPSS Inc., Chicago, IL).

\section{RESULTS}

Clinical parameters. The clinical parameters of the three groups of patients, including early-, late-stage obstructive cholestasis (BA at Kasai operation and BA at OLT) and pediatric control, are listed in Table 1.

Adaptive changes in early-stage and late-stage obstructive cholestasis. In early-stage obstructive cholestasis, the liver showed rapid adaptive changes in that the canalicular transporters, BSEP, MRP2, and MDR3, and the sinusoidal uptake transporters, NTCP and OATP, were downregulated (Fig. 1). Sinusoidal efflux transporters OST $\beta$ was upregulated. The above changes were considered to reduce intracellular bile acid levels and canalicular bile pressure, to protect hepatocytes and cholangiocytes. The downregulation of BSEP was parallel with the lower expression level of its upstream regulator FXR.

In late-stage cholestasis, the FXR and BSEP levels returned to normal. Several other genes showed different adaptive

Table 1. Serum biochemistry in patients with early-and late-stage biliary atresia

\begin{tabular}{lccc}
\hline & BA at Kasai $(n=8)$ & BA at OLT $(n=8)$ & Pediatric control $(n=8)$ \\
\hline Age & $67 \pm 17 \mathrm{~d}$ & $10.50 \pm 3.12 \mathrm{mo}$ & $12.01 \pm 3.86 \mathrm{mo}$ \\
Sex (M:F) & $5: 3$ & 17.4 & $5: 3$ \\
Bilirubin (total) $(\mathrm{mg} / \mathrm{dL})$ & $7.75 \pm 1.60$ & $13.67 \pm 9.77$ & $0.34 \pm 0.15$ \\
Bilirubin (direct) $(\mathrm{mg} / \mathrm{dL})$ & $5.41 \pm 1.32$ & $275.13 \pm 11.49$ & $0.18 \pm 0.07$ \\
AST (U/L) & $181.13 \pm 93.20$ & $197.13 \pm 90.12$ & $70.25 \pm 51.77$ \\
ALT (U/L) & $129.00 \pm 73.75$ & $531.75 \pm 641.89$ & $71.50 \pm 69.74$ \\
GGT (U/L) & $614.00 \pm 654.29$ & $1529.25 \pm 549.14$ & $52.17 \pm 79.61$ \\
ALP (U/L) & $1323.00 \pm 776.89$ & $165.478 \pm 43.545$ & $395.00 \pm 58.35$ \\
Total serum bile acid $(\mu \mathrm{mol} / \mathrm{L})$ & $88.957 \pm 28.697$ & $4.937 \pm 5.427$ \\
Total liver bile acid $(\mu \mathrm{mol} / \mathrm{g})$ & $0.256 \pm 0.106$ & $0.587 \pm 0.094$ & $0.143 \pm 0.032$ \\
\hline
\end{tabular}

BA, biliary atresia; GGT, $\gamma$-glutaryltransferase; OLT, orthotopic liver transplantation. 

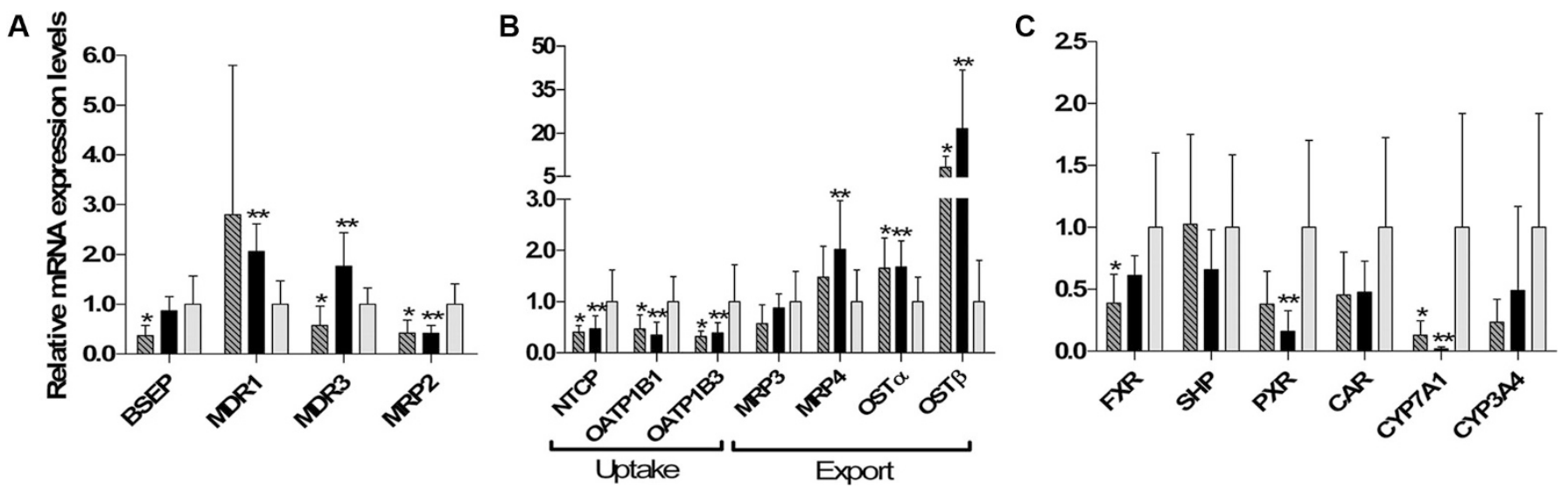

Figure 1. Expression levels of canalicular transporters $(A)$, sinusoidal transporters $(B)$ nuclear receptors and bile acid metabolic enzyme CYP7A1, and CYP3A4 $(C)$ in patients with early-stage (gray bar) and late-stage (black bar) obstructive cholestasis in comparison with pediatric controls (light-gray bar). BA, biliary atresia. Statistically differences are marked as * (BA at Kasai $v s$. pediatric control) or ** (BA at OLT $v s$. pediatric control) when $p<0.05$.

changes, including upregulation of MDR3 (transport phospholipids into bile and protective for bile epithelium) and MDR1, downregulation of MRP2 (decrease bilirubin transport into bile and decrease biliary pressure), inhibition of sinusoidal uptake proteins (NTCP and OATPs) and increased alternative sinusoidal efflux transporters OST $\alpha / \beta$ and MRP4. PXR, the nuclear receptor for bile acid hydroxylation and xenobiotic metabolism, was markedly downregulated at late-stage cholestasis.

The mean levels of nuclear receptor FXR were lower than control in early and late stages $(p=0.025$ and 0.115 , respectively), but only significantly downregulated at early-stage cholestasis. CAR and SHP was unchanged statistically, although there was a trend of lowered CAR levels both at earlyand late-stage cholestasis. The mean levels of PXR were lower than control in early-stage and late-stage cholestasis $(0.38$ and 0.16 of control levels, $p=0.061$ and 0.019 , respectively).

The mean levels of CYP7A1 were lower significantly in early- and late-stage cholestasis compared with control levels, with a marked decreased mean level at late-stage cholestasis ( 0.018 of control levels, $p=0.030$ ). The mean levels of CYP3A4 was also lower in early- and late-stage cholestasis, but without statistical differences $(p=0.098$ and 0.252$)$.

Liver histopathology. Liver pathology revealed small bile plugs in the interlobular bile ducts at early-stage obstructive cholestasis (Fig. 2). At late-stage cholestasis, large bile plugs were noted in the interlobular bile ducts as well as in the large bile ducts. Bile lakes as a result of bile leakage into parenchyma associated with inflammation were seen. These find-

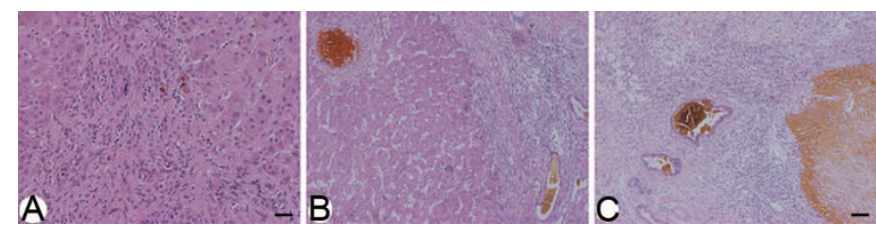

Figure 2. Liver histopathology revealed small bile plugs in the interlobular bile ducts at early-stage obstructive cholestasis (A). At late-stage cholestasis, large bile plugs were noted in the interlobular bile ducts as well as in the large bile ducts. Bile lakes as results of bile leakage into parenchyma associated with inflammatory responses were seen $(B$ and $C$ ). ( $A$ and $B$, magnification: $200 \times$; scale bar: $40 \mu \mathrm{m}$; $C$, magnification: $100 \times$; scale bar: $20 \mu \mathrm{m}$.) ings supported that bile continued to flow into biliary tracts in despite distal obstruction, and were in accordance with the gene expression results shown above.

Nuclear receptors and prognosis with biliary atresia. To investigate whether the expression levels of transporter genes and nuclear receptors had prognostic values at the time of Kasai operation, we analyzed liver samples of 18 patients with $\mathrm{BA}$ at 2 mo of age, and compared the expression levels of BSEP, MDR1, MDR3, OST $\beta$, FXR, PXR, and CAR in BA patients in the good and poor prognosis groups (Table 2). There were no differences in the time of Kasai operation, bilirubin levels, alanine aminotransferase (ALT) levels, and $\gamma$-glutaryltransferase levels at the time of Kasai operation in both groups. We found that patients in the poor prognosis group had significantly lower expression levels of PXR and CAR (Fig. 3). We further divided the patients into high PXR expression levels and low PXR expression levels, using the median expression levels of PXR or CAR as cutoff values. The survival rate and mean survival time for low- and highPXR levels were $25 \%$ vs. $80.0 \% ; 14.3 \pm 2.7$ mo vs. $98.4 \pm$ 12.4 mo, respectively. The survival rate and mean survival time for low and high CAR levels were $33.3 \%$ vs. $77.8 \%$; $27.3 \pm 8.2$ mo vs. $95.9+13.8$ mo, respectively. Survival analysis revealed that the low PXR expression group $(n=8)$ had significantly lower survival rate $(p=0.008)$. Low CAR patients $(n=9)$ also had shorter survival time than high CAR patients $(p=0.041)$. Five of the six patients with both lower PXR and CAR levels all received OLT before $1 \mathrm{y}$ of age (7-11 mo), who were among the patients with worst prognosis.

Immunofluorescent staining. The immunofluorescent staining of BSEP, MDR1, MDR3, and MRP2 in patients with late-stage obstructive cholestasis, in comparison with pediatric and adult controls was shown. The canalicular staining of pediatric hepatocytes in controls and cholestatic liver had reached a similar level and pattern of adult liver. The relative levels of signal intensity in patients with BA in comparison with pediatric control livers confirmed the changes of gene expression levels analyzed by real-time PCR (Fig. 4). BSEP was unchanged in comparison with pediatric control, MRP2 was decreased, and MDR1 and MDR3 were increased. Zonal 
Table 2. Comparisons in the expression levels in patients with biliary atresia (BA) at the time of Kasai operation with good and poor prognosis

\begin{tabular}{lcc}
\hline & Good prognosis $(n=9)$ & Poor prognosis $(n=9)$ \\
\hline Clinical parameters & & \\
Age of Kasai operation & $51 \pm 16$ & $62 \pm 17$ \\
$\quad(\mathrm{~d})$ & $7.07 \pm 2.10$ & $8.36 \pm 2.81$ \\
Bilirubin levels (total) & & \\
$\quad(\mathrm{mg} / \mathrm{dL})$ & $82 \pm 32$ & $107 \pm 81$ \\
ALT (U/L) & $445 \pm 238$ & $623 \pm 455$ \\
GGT (U/L) & & \\
Expression levels of genes & $1.707 \pm 0.591$ & $1.440 \pm 0.660$ \\
BSEP & $0.478 \pm 0.611$ & $0.832 \pm 1.107$ \\
MDR1 & $0.893 \pm 1.032$ & $0.609 \pm 0.423$ \\
MDR3 & $0.332 \pm 0.212$ & $0.441 \pm 0.326$ \\
MRP3 & $3.427 \pm 1.234$ & $5.004 \pm 2.625$ \\
OST $\beta$ & $0.387 \pm 0.180$ & $0.437 \pm 0.201$ \\
FXR & $1.742 \pm 1.141$ & $0.536 \pm 0.422$ \\
PXR & $1.967 \pm 0.796$ & $1.128 \pm 0.634$ \\
CAR & & 0.379 \\
\hline
\end{tabular}

$* p<0.05$.

GGT, $\gamma$-glutaryltransferase.

Relative expression levels normalized to the expression levels of TBP gene are shown.

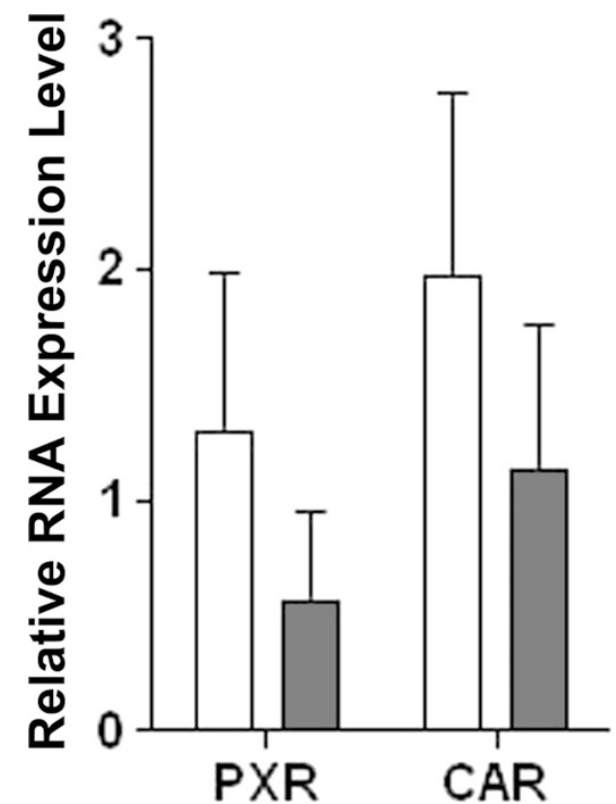

Figure 3. Expression levels of PXR and CAR in patients with biliary atresia at Kasai operation. PXR and CAR levels were found to be lower in patients with poor prognosis (gray bar) than those with good prognosis (white bar) ( $p=0.014$ and 0.025 , respectively).

differences in the expression of canalicular transporters were seen, especially in cholestatic liver, with higher signal intensity at zone 1 and 2, and lesser at zone 3 (data not shown). Colocalization of BSEP with MDR3, MDR1, and MRP2 in the hepatocytes was noted by double-immunofluorescent staining (Fig. 5). MDR1 was also noted to be positive in ductular cells in portal areas, which may represent liver progenitor cells (20). Higher expression levels of OSTs in patients with late-stage BA were confirmed by immunostaining of OST $\beta$ at sinusoidal membrane (Fig. 6).

\section{DISCUSSION}

Our study is the first to delineate chronological changes of hepatocyte transporters and nuclear receptors in human ob- structive cholestasis. The gene expression pattern was quite different between early-stage and late-stage cholestasis (summarized in Fig. 7). During early-stage obstructive cholestasis, the adaptive changes included downregulation of most canalicular and sinusoidal uptake transporters, including BSEP, MDR3, MRP2, NTCP, and OATPs. The downregulation of the major bile transport pathways was probably controlled mainly by downregulation of the key nuclear receptor FXR, because the ligand concentration as reflected by bile acid levels was elevated. The overall response could act to decrease intracellular bile acid levels as well as to reduce biliary pressure. Significant downregulation of PXR, and a trend of lower CAR levels were found, probably represent decreased detoxification capacity in cholestatic liver.

In late-stage obstructive cholestasis, the BSEP returned to normal levels, FXR was also close to the control levels. MDR3, the phospholipids transporter, which acts to protect cholangiocytes, was highly upregulated. MDR1 was also upregulated, probably acting as alternative bile salt exporter in hepatocytes (21), or from the expression of MDR1 in ductular cells or liver progenitor cells (20). At the same time, the sinusoidal transporters OST $\alpha / \beta$ and MRP4 were upregulated to augment bile acid export. Downregulation of CYP7A1 suggested inhibited bile salt synthesis in response to high bile salt levels. The overall responses suggested that in late-stage cholestasis, to compensate for increasingly high levels of intracellular bile acid, bile efflux was bidirectionally through canalicular and sinusoidal membranes, resulting in large bile plugs and bile lakes, as seen in the histopathology of the late-stage liver. The sinusoidal efflux transporters were highly upregulated with a maximal effort to export bile acid into the blood stream, resulting in high serum bile acid levels, and then excreting excessive bile acid through the kidney.

In addition, we found that the localization of the canalicular transporters in pediatric controls and cholestatic liver had reached a similar level and pattern of adult liver, unlike the cytoplasmic localization in fetal liver (19), indicating that the pediatric liver before $1 \mathrm{y}$ of age had obtained a mature canalicular 


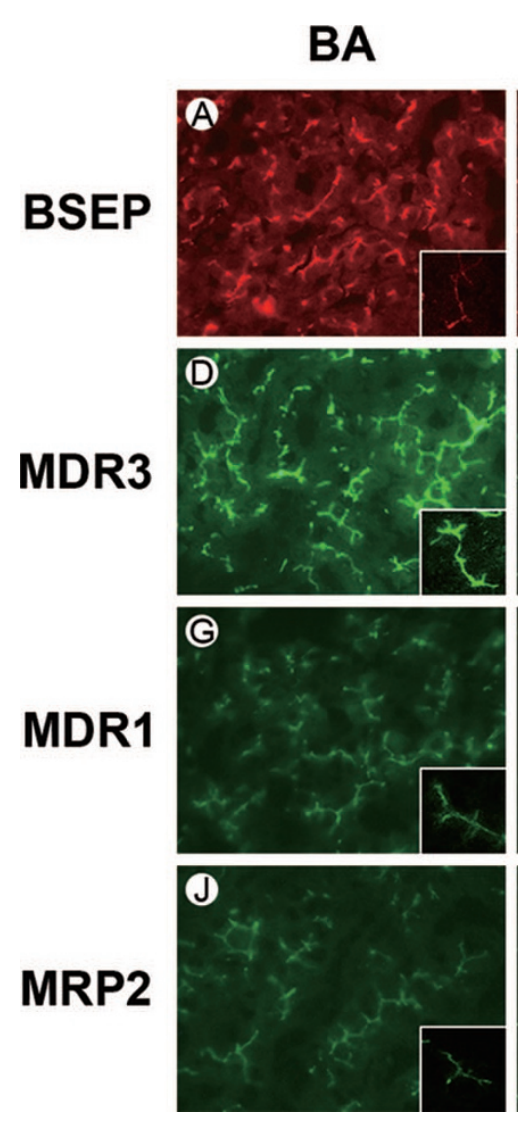

Pediatric
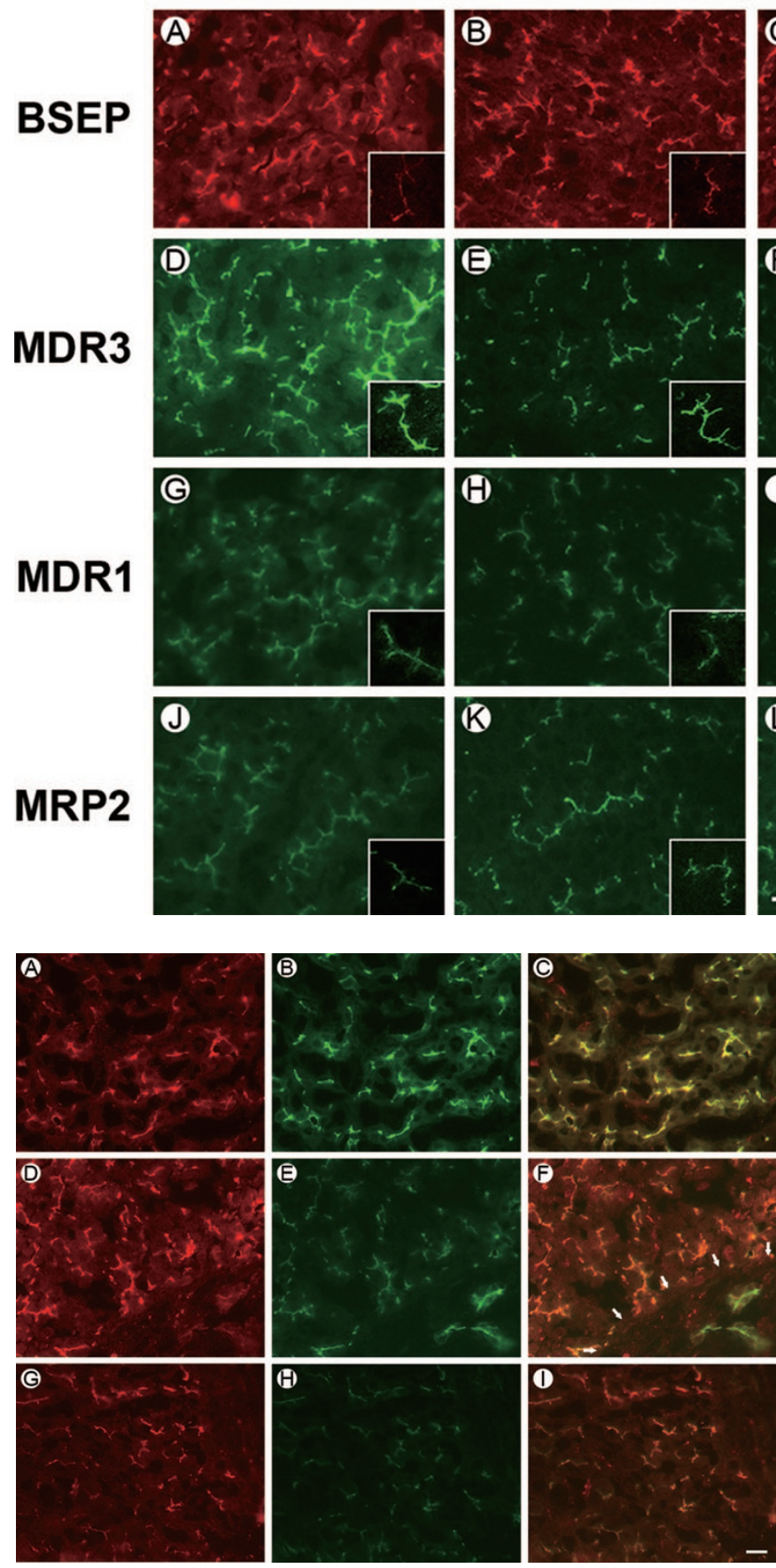

Figure 5. Double fluorescent immunostaining were performed with BSEP (red, $A, D, G$ ) with MDR3, MDR1, and MRP2 (green, $B, E, H$ ). Merged signals (yellow, $C, F, I$ ) at right column were shown. Colocalization of BSEP, MDR3, and MRP2 were shown at canalicular membrane in the hepatocytes in hepatic lobules. MDR1, but not BSEP was also expressed in ductular cells in the portal areas ( $F$, arrow). Scale bar: $20 \mu \mathrm{m}$.

structure. Zonal differences were also noted in the immunoflourescent staining. Hepatocytes in zone 1 receive blood with a high bile salt concentration and therefore are known to be important in bile formation. Our findings supported that during cholestasis, the adaptive changes were most prominent in zone 1 hepatocytes.

Our data were partly in accordance with previous results shown in adult patients with primary biliary cirrhosis, in that
Figure 4. Immunofluorescent staining of the canalicular transporters $\operatorname{BSEP}(A, B, C), \operatorname{MDR} 3(D, E$, $F)$, MDR1 $(G, H, I)$, and MRP2 $(J, K, L)$, from patients with biliary atresia (BA), pediatric controls, and adult controls. Confocal images of the respective transporters in the insets showed the transporters targeted to the canalicular membrane in pediatric and adult livers. BSEP showed similar signal intensity in patients and controls. A marked increase in MDR3 expression was observed in obstructive cholestasis. The MDR1 expression was low in normal liver but increased in cholestatic liver. Signals of MRP2 expression were lower in BA than in pediatric and adult control livers. Scale bar: $20 \mu \mathrm{m}$ and $5 \mu \mathrm{m}$ (insets).
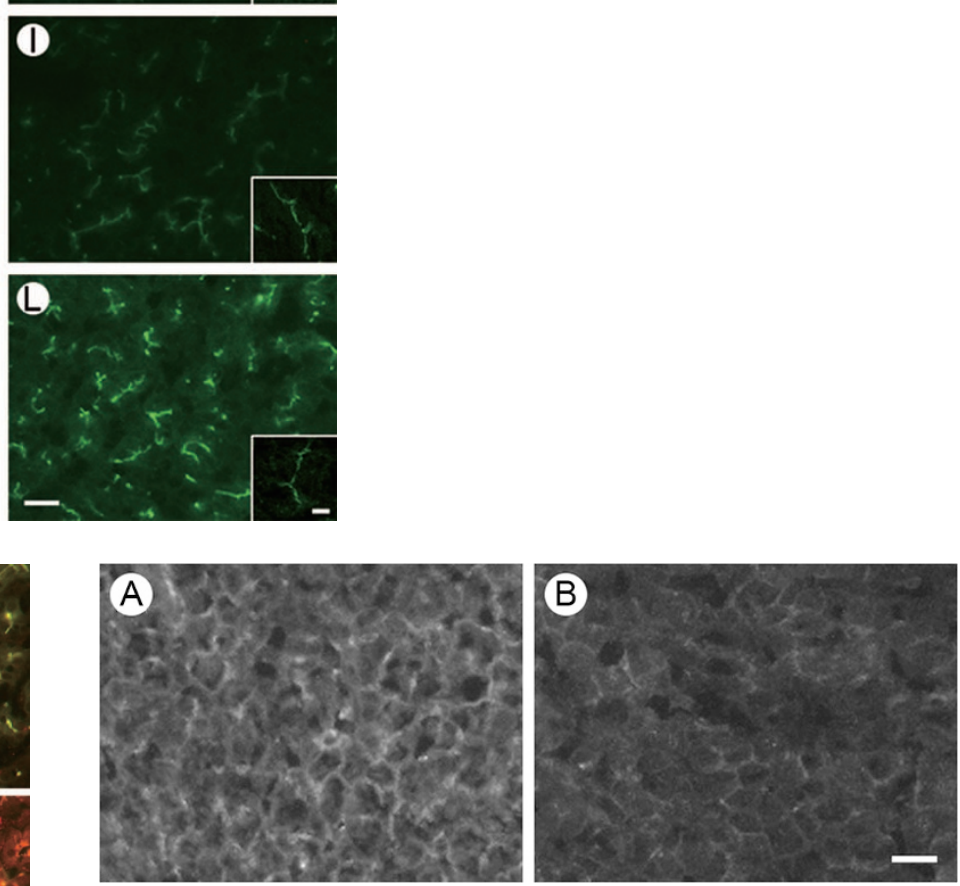

Figure 6. Expression of OST $\beta$ in the sinusoidal membrane was markedly increased in BA $(A)$ than in pediatric control livers $(B)$. Scale bar: $20 \mu \mathrm{m}$.

downregulation of NTCP/OATP, and upregulation of MDR1 and MDR3 at advanced stage; and BSEP maintained in earlystage disease $(15,16)$. Studies in adult obstructive cholestasis patients have shown that BSEP and MRP2 were downregulated, and MRP3 was upregulated $(17,18)$. Our data investigated more genes involving in bile transport and metabolism, as well as the chronological changes of these genes. We have shown that FXR-BSEP were decreased in early-stage and returned toward normal in long-term cholestasis. We did not found upregulation of MRP3 in early and late-stage obstructive cholestasis, which were reported in adult patients $(15,16,18)$. We found that $\operatorname{OST} \alpha / \beta$, the basolateral bile acid efflux proteins $(22,23)$, were highly upregulated in both earlyand late-stage obstructive cholestasis. In this regard, it should be careful to apply the results of transporter changes of one study to other patient population, as different disease pathophysiology, different developmental ages, and species may all affect the adaptive changes in cholestatic liver.

Of note is in late-stage cholestasis, most nuclear receptors were maintained, except PXR. Our study demonstrated that 
A

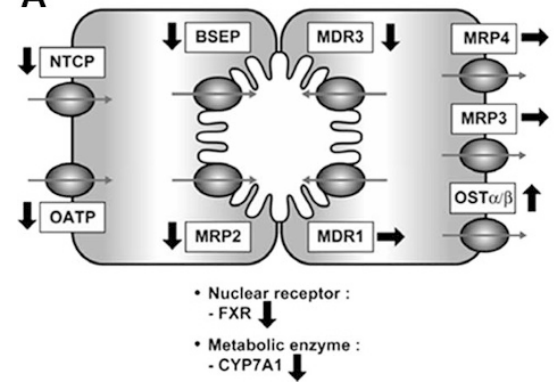

B

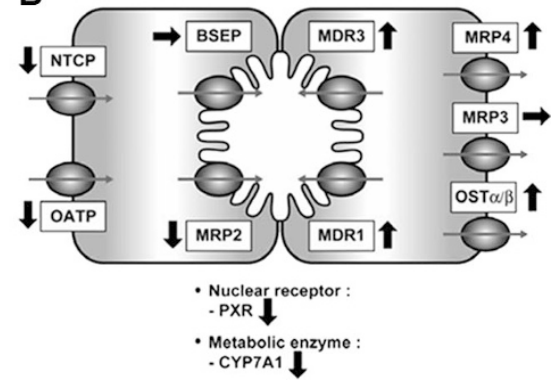

Figure 7. Summary of different adaptive changes of sinusoidal and canalicular transporters, nuclear receptors and CYP genes in pediatric patients with early-stage $(A)$ and late-stage $(B)$ obstructive cholestasis.
PXR was down-regulated significantly in late-stage obstructive cholestasis in BA patients at $1 \mathrm{y}$ of age. In addition, lower PXR and CAR levels were found in the liver of early-stage BA patients with poor prognosis. PXR plays an essential role in the detoxification of the liver, through regulating canalicular and alternative basolateral bile acid excretion, induction of phases I and II bile acid and bilirubin detoxification $(11,13,14)$. It is therefore possible that low levels of PXR would result in poor liver detoxification, and increase the severity of bile acid induced liver toxicity. Interestingly, CAR and PXR agonist had been reported to promote bile acid and bilirubin detoxification and elimination in rats (11). Hepatic damage from bile acid accumulation was aggravated in PXR knockout mice with increased mortality $(8,24)$. Animal studies showed that PXR is antifibrogenic, and that PXR agonist inhibited hepatic stellate cell transdifferentiation and liver fibrosis (25). The ligands for PXR include rifampicin, phenobarbital, ursodeoxycholic acids, which are frequently used to treat cholestatic liver diseases (26). We speculated that patients with lower PXR and CAR might include genetic factors, different inflammatory process, or gene regulation by different infectious pathogens. The pathophysiology of BA is known to be heterogeneous, caused by infection, genetic susceptibility, or different inflammatory responses (4). These genetic or infection-associated factors may affect the regulation of nuclear receptors.

FXR, which is activated by bile acid, regulates BSEP, MDR3, and MRP2. Our data showed that FXR-BSEP was reduced in early-stage cholestasis, which may decrease bile flow. The effect of FXR and BSEP maintenance in late-stage cholestasis is not clear. Inhibition of FXR and BSEP could theoretically be protective in obstructive cholestasis, by reducing bile acid levels and bile flow pressure in the biliary tree $(7,8)$. However, FXR inhibition may also cause hepatocellular necrosis, and the liver injury level and mortality were unchanged $(7,8)$. FXR also takes important roles in lipid and glucose metabolisms (27), as well as normal liver regeneration (28). Therefore, the modulation of FXR in the treatment of obstructive cholestasis deserves further investigation.

Multiple factors involve in the regulation of nuclear receptors on target genes, including the expression levels of nuclear receptors, ligand concentration and activity, cofactors, and other coordinate nuclear receptors (29). Thus, a direct correlation of a single gene with a single regulating nuclear receptor may not be found. Further studies regarding the role of nuclear receptors on the changes of down-stream transporters and genes, as well as the nuclear translocation of these nuclear receptors in the hepatocytes should be investigated before proposed modulation of nuclear receptors could be used as treatment option.

In conclusion, we found that early- and late-stage obstructive cholestasis in human liver showed different adaptive changes in hepatocyte transporter systems. Early responses to obstructive cholestasis resulted in decreased intracellular bile acid uptake and excretion. At late-stage obstructive cholestasis, the overall response decreased bile acid uptake but not canalicular export or biliary pressure. Bile efflux may be through both sinusoidal and canalicular routes. The body tended to maintain homeostasis of FXR-BSEP signaling pathway in long-term cholestasis. Marked downregulation of PXR was found at late-stage cholestasis. PXR and CAR at the time of Kasai operation were associated with prognosis. Treatment aiming at modulating nuclear receptors FXR, PXR, and CAR deserves further investigations.

Acknowledgments. The authors thank Dr. Nazzareno Ballatori, University of Rochester School of Medicine, for kindly providing OST $\beta$ antibody; Nai-Yu Wang, Tien-Jui Lee, Ya-Hui Chen, and Pei-Lin Tsai for technical assistances; and the Department of Medical Research, National Taiwan University Hospital for research support.

\section{REFERENCES}

1. Sherlock S, Dooley J 1997 Diseases of the Liver and Biliary System. 10th ed., Blackwell Science Ltd, London, pp 217-237

2. Fickert P, Zollner G, Fuchsbichler A, Stumptner C, Weiglein AH, Lammert F, Marschall HU, Tsybrovskyy O, Zatloukal K, Denk H, Trauner M 2002 Ursodeoxycholic acid aggravates bile infarcts in bile duct-ligated and Mdr2 knockout mice via disruption of cholangioles. Gastroenterology 123:1238-1251

3. Hung PY, Chen CC, Chen WJ, Lai HS, Hsu WM, Lee PH, Ho MC, Chen TH, Ni YH, Chen HL, Hsu HY, Chang MH 2006 Long-term prognosis of patients with biliary atresia: a 25 year summary. J Pediatr Gastroenterol Nutr 42:190-195

4. Sokol RJ, Mack C, Narkewicz MR, Karrer FM 2003 Pathogenesis and outcome of biliary atresia: current concepts. J Pediatr Gastroenterol Nutr 37:4-21

5. Trauner M, Wagner M, Fickert P, Zollner G 2005 Molecular regulation of hepatobiliary transport systems: clinical implications for understanding and treating cholestasis. J Clin Gastroenterol 39:S111-S124

6. Jansen PL, Sturm E 2003 Genetic cholestasis, causes and consequences for hepatobiliary transport. Liver Int 23:315-322

7. Stedman C, Liddle C, Coulter S, Sonoda J, Alvarez JG, Evans RM, Downes M 2006 Benefit of farnesoid X receptor inhibition in obstructive cholestasis. Proc Natl Acad Sci USA 103:11323-11328

8. Wagner M, Fickert P, Zollner G, Fuchsbichler A, Silbert D, Tsybrovskyy O, Zatloukal K, Guo GL, Schuetz JD, Gonzalez FJ, Marschall HU, Denk H, Trauner M 2003 Role of farnesoid X receptor in determining hepatic ABC transporter expression and liver injury in bile duct-ligated mice. Gastroenterology 125:825-838

9. Ananthanarayanan M, Balasubramanian N, Makishima M, Mangelsdorf DJ, Suchy FJ 2001 Human bile salt export pump promoter is transactivated by the farnesoid X receptor/bile acid receptor. J Biol Chem 276:28857-28865 
10. Denson LA, Sturm E, Echevarria W, Zimmerman TL, Makishima M, Mangelsdorf DJ, Karpen SJ 2001 The orphan nuclear receptor, shp, mediates bile acid-induced inhibition of the rat bile acid transporter, ntcp. Gastroenterology 121:140-147

11. Wagner M, Halilbasic E, Marschall HU, Zollner G, Fickert P, Langner C, Zatloukal K, Denk H, Trauner M 2005 CAR and PXR agonists stimulate hepatic bile acid and bilirubin detoxification and elimination pathways in mice. Hepatology 42:420-430

12. Jung D, Mangelsdorf DJ, Meyer UA 2006 Pregnane X receptor is a target of farnesoid X receptor. J Biol Chem 281:19081-19091

13. Makishima M 2005 Nuclear receptors as targets for drug development: regulation of cholesterol and bile acid metabolism by nuclear receptors. J Pharmacol Sci 97:177183

14. Boyer JL 2005 Nuclear receptor ligands: rational and effective therapy for chronic cholestatic liver disease? Gastroenterology 129:735-740

15. Zollner G, Fickert P, Silbert D, Fuchsbichler A, Marschall HU, Zatloukal K, Denk H, Trauner M 2003 Adaptive changes in hepatobiliary transporter expression in primary biliary cirrhosis. J Hepatol 38:717-727

16. Zollner G, Fickert P, Zenz R, Fuchsbichler A, Stumptner C, Kenner L, Ferenci P, Stauber RE, Krejs GJ, Denk H, Zatloukal K, Trauner M 2001 Hepatobiliary transporter expression in percutaneous liver biopsies of patients with cholestatic liver diseases. Hepatology 33:633-646

17. Yamada T, Arai T, Nagino M, Oda K, Shoda J, Suzuki H, Sugiyama Y, Nimura Y 2005 Impaired expression of hepatic multidrug resistance protein 2 is associated with posthepatectomy hyperbilirubinemia in patients with biliary cancer. Langenbecks Arch Surg 390:421-429

18. Shoda J, Kano M, Oda K, Kamiya J, Nimura Y, Suzuki H, Sugiyama Y, Miyazaki H, Todoroki T, Stengelin S, Kramer W, Matsuzaki Y, Tanaka N 2001 The expression levels of plasma membrane transporters in the cholestatic liver of patients undergoing biliary drainage and their association with the impairment of biliary secretory function. Am J Gastroenterol 96:3368-3378

19. Chen HL, Chen HL, Liu YJ, Feng CH, Wu CY, Shyu MK, Yuan RH, Chang MH 2005 Developmental expression of canalicular transporter genes in human liver. J Hepatol 43:472-477
20. Ros JE, Libbrecht L, Geuken M, Jansen PL, Roskams TA 2003 High expression of MDR1, MRP1, and MRP3 in the hepatic progenitor cell compartment and hepatocytes in severe human liver disease. J Pathol 200:553-560

21. Lam P, Wang R, Ling V 2005 Bile acid transport in sister of P-glycoprotein (ABCB11) knockout mice. Biochemistry 44:12598-12605

22. Ballatori N 2005 Biology of a novel organic solute and steroid transporter, OSTalphaOSTbeta. Exp Biol Med (Maywood) 230:689-698

23. Boyer JL, Trauner M, Mennone A, Soroka CJ, Cai SY, Moustafa T, Zollner G, Lee JY, Ballatori N 2006 Upregulation of a basolateral FXR-dependent bile acid efflux transporter OSTalpha-OSTbeta in cholestasis in humans and rodents. Am J Physiol Gastrointest Liver Physiol 290:G1124-G1130

24. Stedman CA, Liddle C, Coulter SA, Sonoda J, Alvarez JG, Moore DD, Evans RM, Downes M 2005 Nuclear receptors constitutive androstane receptor and pregnane X receptor ameliorate cholestatic liver injury. Proc Natl Acad Sci USA 102:2063-2068

25. Marek CJ, Tucker SJ, Konstantinou DK, Elrick LJ, Haefner D, Sigalas C, Murray GI, Goodwin B, Wright MC 2005 Pregnenolone-16alpha-carbonitrile inhibits rodent liver fibrogenesis via PXR (pregnane X receptor)-dependent and PXR-independent mechanisms. Biochem J 387:601-608

26. Marschall HU, Wagner M, Zollner G, Fickert P, Diczfalusy U, Gumhold J, Silbert D, Fuchsbichler A, Benthin L, Grundstrom R, Gustafsson U, Sahlin S, Einarsson C Trauner M 2005 Complementary stimulation of hepatobiliary transport and detoxification systems by rifampicin and ursodeoxycholic acid in humans. Gastroenterology 129:476-485

27. Zhang Y, Lee FY, Barrera G, Lee H, Vales C, Gonzalez FJ, Willson TM, Edwards PA 2006 Activation of the nuclear receptor FXR improves hyperglycemia and hyperlipidemia in diabetic mice. Proc Natl Acad Sci USA 103:1006-1011

28. Huang W, Ma K, Zhang J, Qatanani M, Cuvillier J, Liu J, Dong B, Huang X, Moore DD 2006 Nuclear receptor-dependent bile acid signaling is required for normal liver regeneration. Science 312:233-236

29. Tirona RG, Kim RB 2005 Nuclear receptors and drug disposition gene regulation. J Pharm Sci 94:1169-1186 